\title{
Enigma of ultraluminous X-ray sources may be resolved by 3D-spectroscopy (MPFS data)
}

\author{
S. Fabrika and P. Abolmasov \\ Special Astrophysical Observatory, Russia \\ fabrika@sao.ru
}

Summary. The ultraluminous X-ray sources (ULXs) were isolated in external galaxies for the last 5 years. Their X-ray luminosities exceed 100-10000 times those of brightest Milky Way black hole binaries and they are extremely variable. There are two models for the ULXs, the best black hole candidates. 1. They are supercritical accretion disks around a stellar mass black hole like that in SS433, observed close to the disk axes. 2. They are Intermediate Mass Black Holes (of 100-10000 solar masses). Critical observations which may throw light upon the ULXs nature come from observations of nebulae around the ULXs. We present results of 3D-spectroscopy of nebulae around several ULXs located in galaxies at 3-6 Mpc distances. We found that the nebulae to be powered by their central black holes. The nebulae are shocked and dynamically perturbed probably by jets. The nebulae are compared with SS433 nebula (W50).

\section{Introduction}

The main properties of the ultraluminous X-ray sources (ULXs) - huge luminosities $\left(10^{39-41} \mathrm{erg} / \mathrm{s}\right)$, diversity of X-ray spectra, strong variability, connection with star-forming regions, their surrounding nebulae. ULXs may be supercritical accretion disks observed close to the disk axis in close binaries with a stellar mass black hole or microquasars (1], 2, 3]). Another idea is that ULXs may be intermediate-mass black holes (IMBHs) with "normal" accretion disks $(4,5)$. It is also possible that ULXs are not homogeneous class of objects.

It was suggested originally by Katz 6 that SS433 being observed close to the jet axis, will be extremely bright X-ray source. Fabrika \& Mescheryakov [1] discussed observational properties of face-on SS433-like objects and concluded that they may appear as a new type of extragalactic X-ray sources. In [7. we discussed possible properties of the funnel in the supercritical accretion disk of SS433. We predicted X-ray spectra and temporal behaviour of the funnel in "face-on SS433" star in application for ULXs. Here we continue to develop this idea and consider nebulae surrounding the ULXs sources.

The main difference between SS433 and other known X-ray binaries is highly supercritical and persistent mass accretion rate $\left(\sim 10^{-4} M_{\odot} / y\right)$ onto the relativistic star (a probable black hole, $\sim 10 M_{\odot}$ ), which has led to the 
formation of a supercritical accretion disk and the relativistic jets. SS433 properties were reviewed recently by 8 .

Similar to SS 433 the ULXs are connected with nebulae. They are frequently located in bubble-like nebulae. New data 9] show that the nebulae are expanding with a velocity $\sim 80 \mathrm{~km} / \mathrm{s}$ (up to $\sim 250 \mathrm{~km} / \mathrm{s}$ ). The nebula sizes are from 20 to a few hundred parsecs, such nebulae are easy for observations even from "megaparsec distances". Here we compare the gas nebula around SS 433 with nebulae of ULXs in Holmberg II, NGC 6946 and IC 342 galaxies observed recently by the Integral-field spectroscopy methods.

\section{Observations and data reduction}

We discuss results of observations on the Russian 6-m telescope with the integral field spectrograph MPFS [10. The integral field unit of $16 \times 16$ square spatial elements covers a region of $16 " \times 16$ " on the sky. Integral field spectra were taken in the spectral range $4000-6800 \AA \AA$ with a seeing 1.0-1.3" (FWHM). Data reduction was made using procedures developed in IDL environment (version 6.0) by V. Afanasiev, A. Moiseev and P. Abolmasov and include all the standard steps.

\section{Nebulae surrounding ULXs and SS433}

The object SS 433 is surrounded by the elongated radio nebula W 50, which was produced (or distorted) by SS433 jets due to the jet interaction with interstellar medium [11. The radio emission is synchrotron, relativistic electrons appear at the jet deceleration. Bright optical filaments are observed [12] in places of the jets termination, they radiate in $\mathrm{HI}$, [OI], [NII], [SII] lines. Optical filaments in the bipolar nebula are located at $\pm 0.5^{\circ}$ or $\pm 50 p c$ from SS433. A total energy of the nebula is $E_{k} \sim 2 \cdot 10^{51} \mathrm{erg}$ [12], which corresponds to the jet kinetic luminosity $L_{k} \sim 3 \cdot 10^{39} \mathrm{erg} / \mathrm{s}$ for 20000 years. The observed velocity dispersion in the filaments is $\sim 50 \mathrm{~km} / \mathrm{s}$, however $[\mathrm{NII}] / \mathrm{H} \alpha$ line ratio corresponds to dispersion $\sim 300 \mathrm{~km} / \mathrm{s}$ [12. SS433 is an edge-on system, $i=79^{\circ}$. If one takes into account this factor, the velocity dispersion may reach $250-300 \mathrm{~km} / \mathrm{s}$. The ULXs nebulae studied by us, have about

the same sizes, the same line luminosities and about the same total energy budget $10^{51-52}$ erg [13].

In Fig 1 we present emission line maps of the nebula MF 16 (" a peculiar SN remnant"), surrounding ULXs in the galaxy NGC 6946. In [OIII] $\lambda 4959,5007$ lines we found a radial velocity gradient across the nebula in East-West direction. In later observations using spectrograph SCORPIO [14] in a longslit mode we confirmed this gradient. The radial velocity gradient reaches $100 \mathrm{~km} / \mathrm{s}$ across the whole nebula $(20 \mathrm{pc})$. 

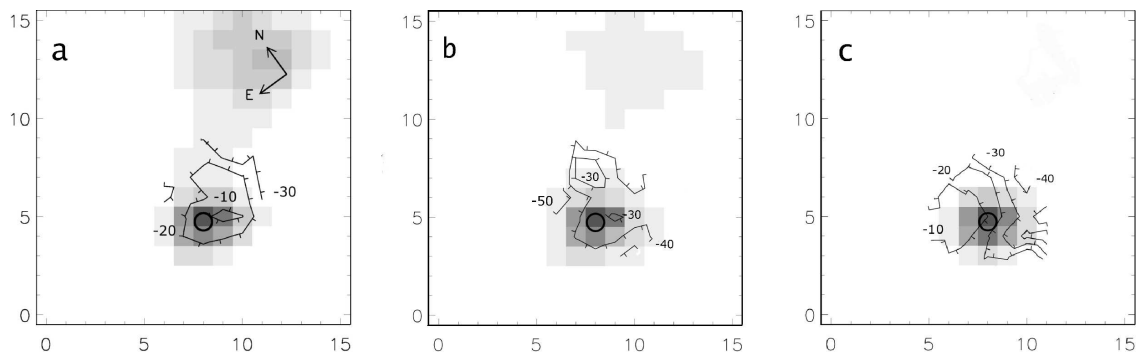

Fig. 1. $\mathrm{H} \alpha$ (a), [SII] $\lambda 6717,6734$ (b) and [OIII] $\lambda 4959,5007$ (c) $15 \times 15$ " maps of MF 16 nebula surrounding the NGC 6946 ULX-1. Circles show location of the X-ray source from GHANDRA's data. Equal radial velocty lines are shown. Marks show a direction of increasing of the velocity absolute value.
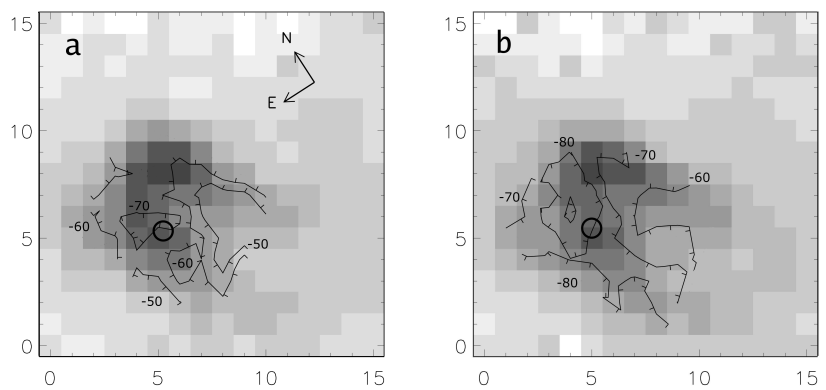

Fig. 2. $\mathrm{H} \alpha$ (a) and $[\mathrm{SII}] \lambda 6717,6734$ (b) maps of the nebula surrounding the IC 342 ULX-1. Designation are the same as in Fig 1.

In Fig 2 we present results of observations of the nebula surrounding the ULX-1 in the galaxy IC 342 using the same device. In this nebulae we have also detected a radial velocity gradient as a whole expansion of the nebula $\pm 20 \mathrm{~km} / \mathrm{s}$. This nebula is not bright because of strong light absorption in direction of IC 342 .

Fig 3 presents results of observations of Holmberg II ULX-1 nebula with the MPFS and SCORPIO spectrograps. Bright He II $\lambda 4686$ emission line is observed in this nebula. We have also detected a radial velocity gradient $\pm 50 \mathrm{~km} / \mathrm{s}$ on a spatial scale $\pm 50 \mathrm{pc}$ in the nebula [15]. The radial velocity across the nebula has been measured in more details in observations with SCORPIO.

In all three studied ULXs nebulae diagnostic line ratios indicate collisional excitation of the gas (see 14 for more details). We obtain two more conclusions which may be principal for understanding of the ULXs:

1 . The radial velocity gradient of $50-100 \mathrm{~km} / \mathrm{s}$ on spatial scales $20-100 \mathrm{pc}$ testify that the nebulae are dynamically perturbed. The IMBHs can not perturb the interstellar gas on such big scales, the capture Bondi radii are not greater than $0.1 \mathrm{pc}$. These nebulae can not be SNRs, they are too big and 

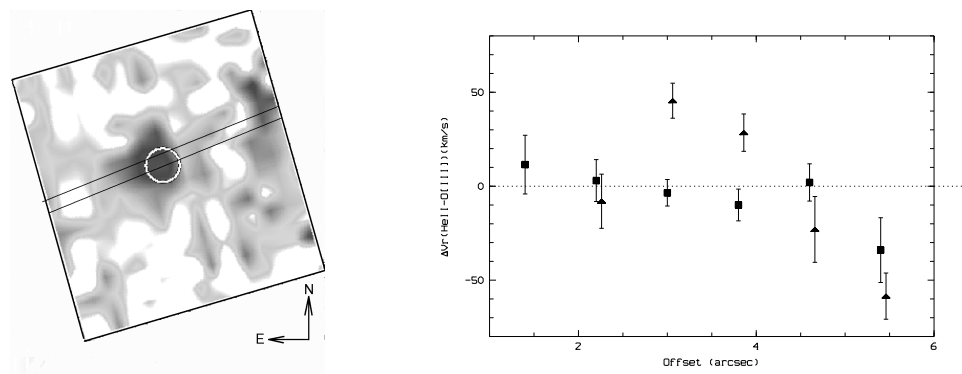

Fig. 3. HeII $\lambda 4686$ map of the nebula surrounding the Holmberg II ULX-1 (left). Slit positions in LS-spectroscopy are also shown. Radial velocities (right) of this line measured along the slits relative to the [OIII $] \lambda 5007$ line are shown by squares for the upper slit and by triangles for the bottom slit.

energetic, they do not satisfy to standard relations for SNRs. It is very probable that the nebulae are powered by stellar wind or jets like that it is in SS 433.

2. For explanation of luminosities in high excitation lines and of the whole spectrum we need an additional source of hard UV radiation [14]. The source luminosity is the same huge $\left(\sim 10^{40} \mathrm{erg} / \mathrm{s}\right)$ as that in X-rays.
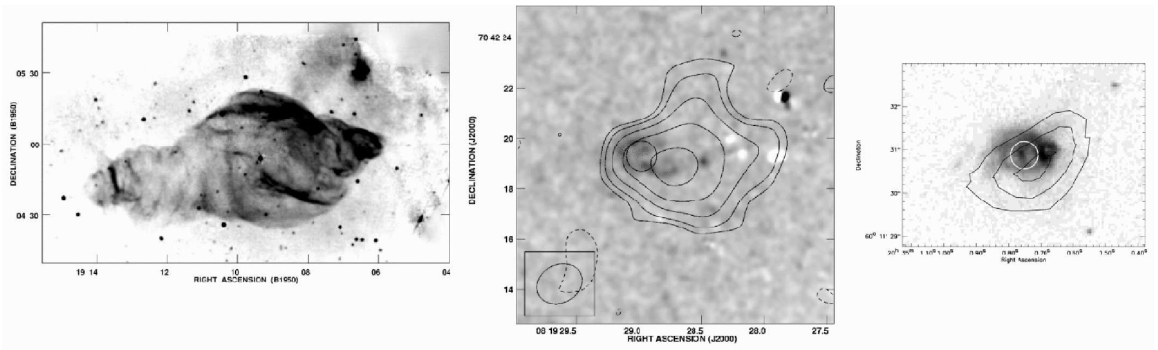

Fig. 4. Three nebulae in the same scale in parsecs. VLA image of W50 11 with SS 433 in center, left; Holmberg II ULX-1 in HST HeII image with VLA isophotes, middle [16] and NGC 6946 ULX-1 in HST H $\alpha+$ [SII] image with VLA isophotes [17, right. Circles show X-ray Chandra positions.

In Fig. 4 we present in the same linear scale the nebula W 50 together with nebulae surrounging ULXs in Holmberg II and NGC6946 galaxies 15, 13. The nebulae in Holmberg II and NGC 6946 have circle-like features in the line-images. In both cases the radio sources are shifted to a brighter circlelike feature. The radio sources are not resolved. In the both cases the part of the nebulae coinciding with radio source is approaching, the opposite part is receding [15], [13]. At some imagination one may conclude that the nebulae around these two ULXs are face-on versions $\left(i=10^{\circ}-30^{\circ}\right)$ of the SS 433 
nebula. We need to continue observations to take more representative sample of nebulae connected with ULXs.

\section{Acknowledgements}

This work has been supported by the RFBR grant 04-02-16349 and RFBR/JSPC grant N 05-02-19710. The authors are grateful to the SOC and LOC of the Workshop "Science Perspectives for 3D Spectroscopy" for support.

\section{References}

1. S. Fabrika, Mescheryakov: In: Galaxies and their Constituents at the Highest Angular Resolution, IAU Symp. N205, ed by R.T. Schilizzi, (Manchester, United Kingdom 2001) p. 268

2. A.R. King, M.B. Davies, M.J. Ward, G. Fabbiano, M. Elvis: ApJ (Letters) 552, L109 (2001)

3. E. Koerding, H. Falcke, S. Markoff, R. Fender: Astron. Gesells. Meet. Abstr. 18, $176(2001)$

4. E.J.M. Colbert, R.F. Mushotzky: ApJ 519, 89 (1999)

5. J.M. Miller, A.C. Fabian, M.C. Miller: ApJ (Letters) 614, L117 (2004)

6. J.J. Katz: ApJ 317, 264 (1987)

7. S. Fabrika, S. Karpov, P. Abolmasov, O. Sholukhova: In Populations of High Energy Sources in Galaxies, IAU Symposium N230, ed by E.J.A. Meurs \& G. Fabbiano, 2006 in press

8. S. Fabrika: Astrophysics and Space Physics Reviews, 12, 1 (2004)

9. M. Pakull: In Populations of High Energy Sources in Galaxies, IAU Symposium N230, ed by E.J.A. Meurs \& G. Fabbiano, 2006 in press

10. V.L. Afanasiev, S.N. Dodonov, A.V. Moiseev: In Stellar dynamics: from classic to modern, ed by Osipkov L.P., Nikiforov I.I. (Saint Petersburg 2001) p. 103

11. G.M. Dubner, M. Holdaway, W.M. Goss, I.F. Mirabel: AJ 116, 1842 (1998)

12. W.J. Zealey, M.A. Dopita, D.F. Malin: MNRAS 192, 731 (1980)

13. S. Fabrika, P. Abolmasov, O. Sholukhova: 2006, in preparation

14. P. Abolmasov, S. Fabrika, O. Sholukhova: 2006, in preparation

15. I. Lehmann, T. Becker, S. Fabrika et al: A\&A 431, 847 (2005)

16. N.A. Miller, R.F. Mushotzky, S.G. Neff: ApJ (Letters) 623, L109 (2005)

17. S.D. van Dyk, R.A. Sramek, K.W. Weiler et al: ApJ (Letters) 425, L77 (1994) 